\title{
2D Computer Model of a Non-Stationary Corona from an Infinite Grating Composed of Parallel Grounded Wires in a Thundercloud Electric Field
}

\author{
M. S. Mokrov, Yu. P. Raizer \\ Ishlinsky Institute for Problems in Mechanics RAS, Moscow, 119526, Russia \\ mmokrov@gmail.com
}

\begin{abstract}
This paper deals with a non-stationary corona developing in free space from a plane-parallel infinite periodic system of grounded wires suspended at the same height in a growing uniform thundercloud electric field. The discharge is described by the continuity equation for positive ions and electrostatic equation for the electric field induced by wire charges and ion space charge. These equations were solved using a two-dimensional computer program, which was developed in this study and then used to calculate the corona current, the distribution of the space charge generated by the corona, and the potential distribution in space surrounding the wires. The obtained characteristics of the corona may be of interest for the purpose of increasing the reliability of lightning protection wires.
\end{abstract}

Keywords: corona discharge, numerical simulation, atmospheric electricity.
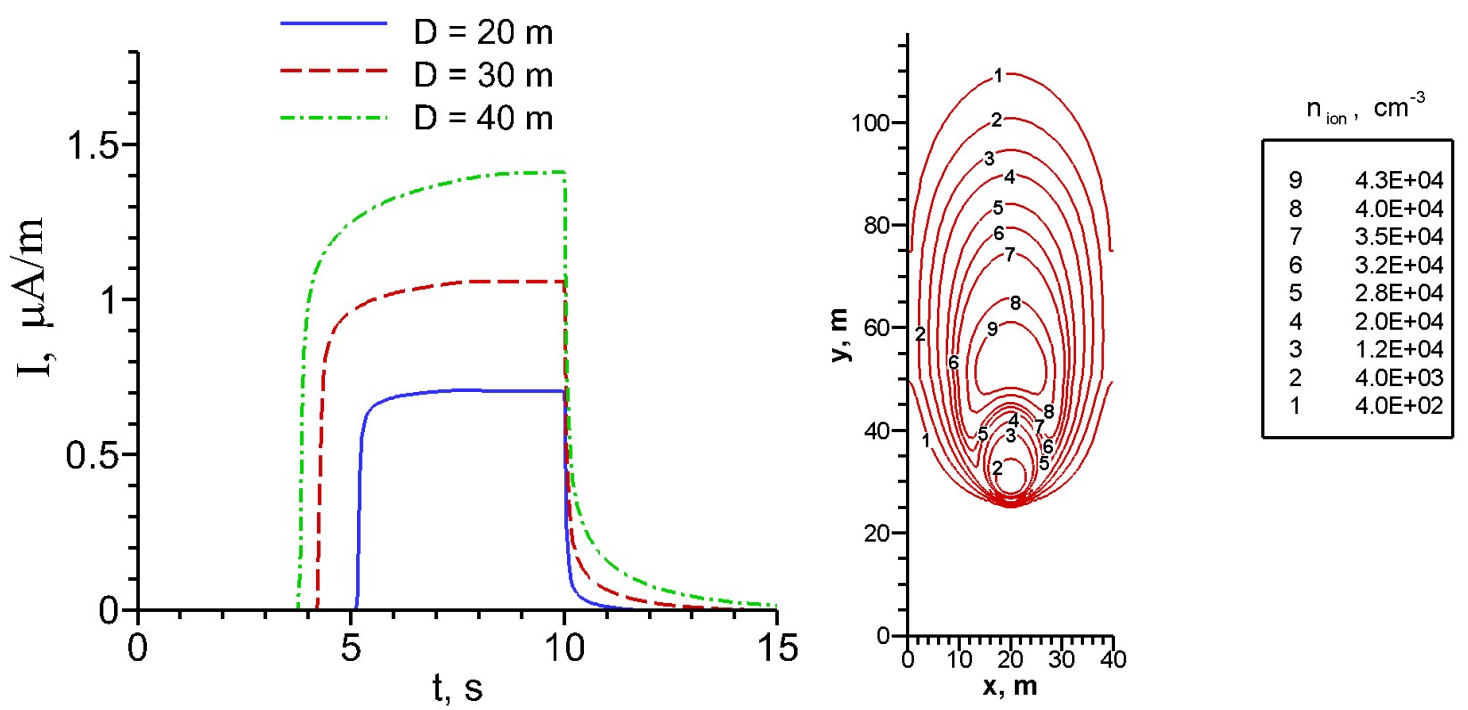

(Left figure) Evolution in time of the corona current per unit length of a grounded wire in an infinite periodic multi-wire system with wires of radius $r_{0}=1 \mathrm{~cm}$, suspended at a height $h=30 \mathrm{~m}$. The period of the wire grating varies from $D=20 \mathrm{~m}$ to $40 \mathrm{~m}$. The thundercloud electric field rises linearly from zero to $E_{0 \max }=400 \mathrm{~V} \mathrm{~cm}^{-1}$ for $\tau=10 \mathrm{~s}$ and then is equal to $E_{0 \max }$ at $t>\tau$. (Right figure) Contour lines of the ion density $n$ (in $\mathrm{cm}^{-3}$ ) injected by a coronating infinite periodic multi-wire system with wires of radius $r_{0}=1 \mathrm{~cm}$, suspended at a height $h=30 \mathrm{~m}$. The distance between wires is $D=40 \mathrm{~m}$ 


\title{
Двумерная модель нестационарного коронного разряда от периодической плоскопараллельной системы заземленных проводов в электрическом поле грозового облака
}

\author{
М. С. Мокров, Ю. П. Райзер \\ Институт проблем механики им. А.Ю. Ишлинского Российской академии наук, \\ Россия, Москва, 119526, проспект Вернадского, д. 101-1, \\ mmokrov@gmail.com
}

\begin{abstract}
Аннотация
Рассмотрен теоретически нестационарный коронный разряд, развивающийся в свободном пространстве от плоскопараллельной бесконечной периодической системы заземленных проводов, подвешенных на одинаковой высоте и находящихся в нарастающем однородном электрическом поле грозового облака. Разряд описывается системой уравнений для плотности положительных ионов и уравнением Пуассона для электрического поля. Для решения этих уравнений разработана двумерная компьютерная программа, на основе которой рассчитаны коронный ток, распределения объемного заряда, генерируемого короной, и распределения потенциала в окружающем провода пространстве. Полученные характеристики короны могут представлять интерес для повышения надежности многотросовой молниезащиты заземленных объектов.
\end{abstract}

Ключевые слова: коронный разряд, численное моделирование, атмосферное электричество.

\section{1. Введение}

Нестационарная корона, возникающая в грозовую погоду у острых выступов заземленных объектов, подробно изучалась за последние 20 лет, см. [1-12] и ссылки. Практическим стимулом этих работ, в основной массе теоретических и расчетных, является наблюдаемое снижение вероятности развития встречного молниевого разряда от заземленного объекта в условиях, когда около объекта успевает развиться коронный разряд $[1,2,13-15]$.

В результате многолетних исследований группы ученых из Энергетического Института им. Г. М. Кржижановского, ИПМех РАН и МФТИ (см. [1-3, 9-11] и ссылки) на основе приближенной квазиодномерной модели короны впервые были детально изучены характеристики нестационарного коронного разряда от заземленных электродов в различных условиях и даны оценки влияния коронного разряда на притяжение молний к высоким объектам. Эти расчеты выполнены для системы заземленных сферических электродов и стержней с полусферической головкой. Позднее, в $[6,8]$ та же задача решалась на основе более детальной двумерной модели нестационарной короны.

Большой интерес для изучения как природных явлений $[9,10,16-18]$, так и новых практических подходов в молниезащите $[4,13,19,20]$ представляют характеристики нестационарного коронного разряда в условиях, когда одновременно коронируют несколько или, в предельном случае, бесконечное множество заземленных проводников. Нестационарная корона от многоэлектродной системы такого рода для случая одинаковых сферических проводников впервые теоретически рассматривалась в [9, 10]. 
Целью данной работы является разработка и тестирование двумерной компьютерной модели нестационарной короны от многоэлектродной системы горизонтальных заземленных проводов, которые являются прототипами молниезащитных тросов. Компьютерная модель позволяет рассчитать погонный ток короны с каждого провода, распределение электрического поля, созданного зарядами проводов и объемным зарядом ионов, внедренным короной от проводов в окружающее пространство.

Компьютерная модель короны, разработанная в этой работе, строится путем обобщения ранее развитой двумерной методики, примененной нами в [7] для расчета короны от одиночного провода в грозовых условиях. В общем случае, когда коронируют несколько проводов, подвешенных на разных высотах, расчет становится очень громоздким, поскольку каждый провод в компьютерной программе необходимо учитывать индивидуально.

Поэтому здесь будет рассматриваться упрощенная задача о короне от бесконечной плоскопараллельной периодической решетки проводов в электрическом поле грозового облака. Предполагается, что все провода тождественны, подвешены на одинаковой высоте $h$, а соседние провода находятся на одинаковом расстоянии друг от друга. Возможным искривлением проводов вдоль его длины под действием собственного веса пренебрегается. В силу периодичности системы достаточно рассмотреть корону от одного провода, а действие остальных проводов заменить периодическими граничными условиями. Такое упрощение, сводящее рассмотрение короны от многоэлектродной системы к короне от одного провода, позволяет сравнительно просто получить численное решение задачи о короне в многоэлектродной системе. Аналогичным образом поступали авторы [21], когда рассматривали обтекание бесконечной решетки цилиндров и сводили эту задачу к задаче об обтекании одного цилиндра с периодическими граничными условиями. Решение задачи о короне от бесконечной периодической системы заземленных проводов, подвешенных над землей, послужит отправной точкой при решении более реалистичной задачи, когда число проводов в системе конечно.

\section{2. Постановка задачи и уравнения}

Рассматривается процесс коронирования бесконечной системы длинных заземленных горизонтальных проводов (молниезащитных тросов) радиуса $r_{0}$, подвешенных над землей на высоте $h$ и находящихся в однородном электрическом поле грозового облака $\mathbf{E}_{0}$. Расстояние между соседними проводами равно $D$. Облако предполагается заряженным отрицательно, так что поле $\mathbf{E}_{0}$ направлено вертикально вверх, а корона от многоэлектродной системы заземленных тросов положительна. В грозовых условиях $\mathbf{E}_{0}$ сначала растет со временем по мере накопления зарядов грозовой ячейки, а затем стабилизируется. Если на последней стадии происходит разряд молнии, то в результате частичной нейтрализации облачного заряда поле $\mathbf{E}_{0}$ может резко снизиться. Однако, в дальнейшем, как правило, $\mathbf{E}_{0}$ нарастает вновь, и все повторяется. Мы будем рассматривать коронный процесс на стадии роста $\mathbf{E}_{0}$.

Наблюдаемое изменение грозового поля $\mathbf{E}_{0}$ обычно аппроксимируют либо линейной функцией вида

$$
\begin{array}{ll}
E_{0}(t)=E_{0 \max } \frac{t}{\tau}, \quad t<\tau ; \\
E_{0}(t)=E_{0 \max }, \quad t \geq \tau,
\end{array}
$$

где $\tau$ - характерное время нарастания грозового поля, либо следующей релаксационной зависимостью:

$$
E_{0}(t)=E_{0 \max }\left[1-\exp \left(-\frac{t}{\tau}\right)\right]
$$


Согласно электростатике, поле $E\left(r_{0}\right)$ у поверхности заземленного проводника радиуса $r_{0} \ll h$ усиливается по сравнению с полем $\mathbf{E}_{0}$ в окружающем пространстве. В некоторый момент $t_{i}$ у проводов зажигается корона. Это происходит, когда $E\left(r_{0}\right)$ превышает порог зажигания короны $E_{\text {cor }}$, который дается известной формулой Пика

$$
E_{\text {cor }}\left(r_{0}\right)=31\left(1+0.308 r_{0}^{-1 / 2}\right) \kappa \mathrm{B} / \mathrm{cm}, \quad r_{0}[\mathrm{~cm}]
$$

Когда число проводов в системе бесконечно, и каждый из них находится в равном положении по отношению к остальным, корона зажигается от всех проводов одновременно. В ходе дальнейшей эволюции поля в окружающее пространство внедряется объемный заряд положительных ионов. Ионы рождаются в узком ионизационном слое около проводов. Ионное облако растет, в особенности вверх, в сторону грозового облака. Из-за присутствия земли и вертикального внешнего поля, этот процесс двумерен. Распределения электрического поля и объемного заряда в пространстве зависят от горизонтальной $x$ и вертикальной $y$ координат в плоскости, перпендикулярной проводам (см. схему расчетной области на рис. 1). Поскольку число проводов бесконечно, и они находятся в равном положении, рассматривается корона от одного провода, а действие остальных заменяется граничными условиями симметрии. От координаты $z$, направленной вдоль проводов, в идеализированном случае горизонтальных проводов ничего не зависит, т.е. решается плоская двумерная задача.

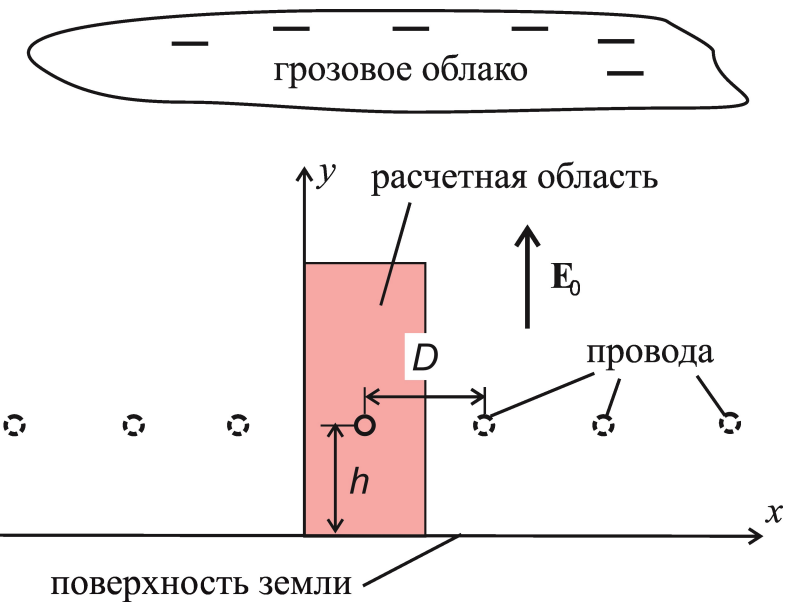

Рис. 1. Схематическое изображение бесконечной периодической системы одинаковых проводов, находящихся во внешнем поле грозового облака $\mathbf{E}_{0}$. Расчетная область выделена цветом

Коронный процесс описывается уравнением непрерывности для плотности ионов $n_{\text {ion }}$ и уравнением электростатики для вектора электрического поля $\mathbf{E}^{\prime}$, созданного зарядами проводов и объемным зарядом ионов, генерируемых короной. Ионы движутся в суммарном электрическом поле $\mathbf{E}=\mathbf{E}^{\prime}+\mathbf{E}_{0}$. Предполагается, что все ионы одного сорта. Система решаемых уравнений имеет вид

$$
\begin{gathered}
\frac{\partial n_{\text {ion }}}{\partial t}+\operatorname{div}\left[n_{\text {ion }} \mu\left(\mathbf{E}^{\prime}+\mathbf{E}_{0}\right)\right]=0, \\
\operatorname{div} \mathbf{E}^{\prime}=\frac{e n_{\text {ion }}}{\varepsilon_{0}}, \quad \mathbf{E}^{\prime}=-\nabla \varphi^{\prime},
\end{gathered}
$$

где $\mu$ - подвижность ионов; $\varepsilon_{0}$ - диэлектрическая проницаемость вакуума; $\varphi^{\prime}-$ потенциал поля $\mathbf{E}^{\prime}$. В расчетах принято $\mu=1.5 \mathrm{~cm}^{2} /(\mathrm{B} \cdot \mathrm{c})$. Потенциал грозового поля отсчитывается от 
земли, где $y=0, \varphi^{\prime \prime}=-E_{0} y$. В компьютерной программе вместо (5) в действительности решается уравнение Пуассона для потенциала $\varphi^{\prime}$

$$
\frac{\partial^{2} \varphi^{\prime}}{\partial x^{2}}+\frac{\partial^{2} \varphi^{\prime}}{\partial y^{2}}=-\frac{e n_{\text {ion }}}{\varepsilon_{0}}
$$

Граничные условия к уравнениям (4), (6) имеют следующий вид. На земле принимаем, что $\varphi^{\prime}=0$. Поскольку провода заземлены, суммарный потенциал проводов $\varphi^{\prime}+\varphi^{\prime \prime}=0$, отсюда $\varphi^{\prime}=E_{0}(t) h$. Ситуация отчасти аналогична случаю нагруженного провода с потенциалом $U(t)=E_{0}(t) h$. После зажигания короны поле на проводе автоматически удерживается равным полю зажигания (3), $E\left(r_{0}\right)=E_{\text {cor }}$. Такое граничное условие обычно принимается в теории бесстримерной короны [1-12].

Поскольку задача симметрична относительно прямых $x=0$ и $x=D$, полагаем, что вдоль этих прямых выполнены условия $\partial n_{\text {ion }} / \partial x=\partial \varphi^{\prime} / \partial x=0$. Граничное условие для потенциала $\varphi^{\prime}$ на верхней границе прямоугольной расчетной области, $y_{\max }$, получается из требования, что верхняя граница фронта объемного заряда ионов не достигает верхней границы расчетной области за все время моделирования. Другими словами, потенциал $\varphi^{\prime}$ при $y=y_{\max }$ находится из условия сшивки двух решений: искомого решения уравнения Пуассона при $y \leq y_{\max }$ и решения уравнения Лапласа при $y \geq y_{\max }$.

Корона зажигается, когда выполнено условие

$$
E\left(r_{0}\right)=E_{\text {cor }},
$$

т.е. когда поле на проводе превысит порог $E_{\text {cor }}$, который дается формулой (3). Начиная с момента зажигания короны, рассчитывается распределение плотности ионов в окружающем провод пространстве $n_{\text {ion }}(x, y, t)$ и, в частности, в первой счетной ячейке у провода, $n_{\text {ion }}\left(r_{0}, t\right)$. С величиной $n_{\text {ion }}\left(r_{0}, t\right)$ связан коронный ток на единицу длины провода, который находится как

$$
I(t)=2 \pi r_{0} e n_{\text {ion }}\left(r_{0}, t\right) \mu E_{\text {cor }}, \quad n_{\text {ion }}=n_{\text {ion }}\left(r_{0}, t\right)
$$

В ходе всей дальнейшей эволюции плотность ионов в первой ячейке $n_{\text {ion }}\left(r_{0}, t\right)$ находится из условия, чтобы поле на проводе $E\left(r_{0}\right)$ удовлетворяло условию (7). При расчете $n_{\text {ion }}\left(r_{0}, t\right)$ пренебрегается малыми вариациями поля $E\left(r_{0}\right)$ на проводе. Эти изменения $E\left(r_{0}\right)$ связаны с электростатической индукцией в грозовом поле, а также в поле, создаваемом зарядами соседних проводов и отражениями зарядов проводов в земле.

\section{3. Численный метод}

Коронный процесс рассчитывается в области $0 \leq x \leq D, 0 \leq y \leq y_{\max }$ (см. рис. 1). Предполагается, что верхняя граница фронта ионного облака не достигает горизонтальной прямой $y=y_{\max }$ за все время моделирования. В расчетах принималось, что $y_{\max }=150 \div 250$ м. В расчетной области строится блочная сетка, состоящая из одного квадратного блока, содержащего провод и прямоугольных блоков, число которых в общем случае равно двум. В квадратном блоке с проводом строится сетка $\mathrm{O}$ - типа, линии которой охватывают провод. Методика построения этой сетки [22] основана на численном решении двух двумерных уравнений эллиптического типа для координат $x, y$ узлов сетки. В остальных блоках сетки вводится прямоугольная сетка, равномерная вдоль направления $x$ и неравномерная вдоль $y$. Пример построенной сетки с числом контрольных объемов равным 11130 дан на рис. 2 .

Длина стороны квадратного блока сетки, содержащего провод, равна расстоянию между проводами $D$. При каждом изменении $D$ сетка генерируется заново. При принятом числе узлов сетки, процедура генерации сетки занимала не более десяти секунд, что не является слишком затратным. Заметим, что поскольку ионы движутся в основном вертикально 
вверх, размах расчетной области по вертикали должен быть существенно большим, чем по горизонтали. Это и оправдывает введение многоблочной сетки.

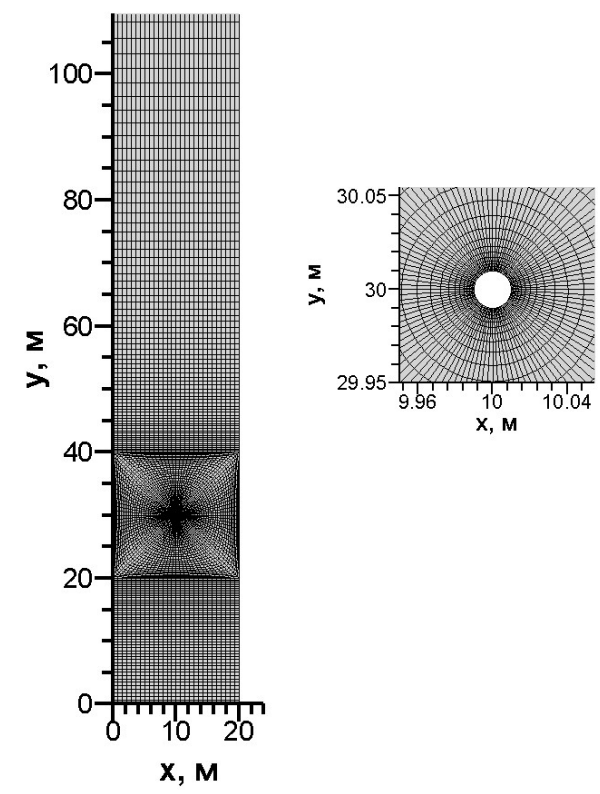

Рис. 2. Пример расчетной сетки. Высота подвеса проводов $h=30$ м, расстояние между проводами $D=20$ м, радиус проводов $r_{0}=1 \mathrm{~cm}$

Отметим, что основная трудность решаемой задачи состоит в сильном различии масштабов физических процессов. В самом деле, за характерное время нарастания грозового поля $\tau \sim 10$ с верхняя граница фронта ионного заряда продвигается вверх, к грозовому облаку, на расстояния порядка сотни метров, что на четыре порядка превышает радиус коронирующих проводов, $r_{0} \sim 1$ см. Расчет короны должен вестись на сетке с шагом, увеличивающимся по мере удаления от провода; иначе выполнить расчет на персональном компьютере было бы нереально. Но такое укрупнение шага сетки приводит к размытию границы фронта расширяющегося облака объемного заряда.

Для упрощения вычислений, вместо использования адаптивной сетки, сгущающейся вблизи фронта, все расчеты проведены на фиксированной сетке, подобной той, которая изображена на рис. 2. Оправданием этому служит тот факт, что, как показывают проведенные в [7] расчеты, равномерное измельчение сетки, приводящее к лучшему пространственному «разрешению» фронта, мало влияет на коронный ток, количество внедренного заряда и распределение электрического поля в коронном облаке.

Уравнения (4) и (6) приводятся к конечно-разностному виду методом конечного объема [23]. Для уравнения непрерывности (4) используется «upwind» схема первого порядка точности по пространству и неявная схема Эйлера с первым порядком точности по времени. В результате для значений плотности ионов получается система линейных уравнений, в которой плотности ионов в пяти соседних ячейках связаны между собой. После применения метода конечного объема к уравнению для потенциала (6), требуется вычислить градиенты потенциала в центрах граней ячеек. Для этого используется «метод интегрирования по траекториям» из [24]. В результате получается линейная система уравнений для потенциала; при этом неизвестный потенциал в текущей ячейке связан с потенциалами в девяти соседних точках. Линейные уравнения для $n_{\text {iоn }}$ и $\varphi^{\prime}$, полученные в результате аппроксимации (4) и (6), дополненные граничными условиями, решаются методом релаксации с прогонкой по линиям на каждом шаге по времени. Распределения $n_{\text {ion }}$ и $\varphi^{\prime}$, рассчитанные в различных блоках сетки, сшиваются с помощью линейной интерполяции. 
Подчеркнем, что предположение $\varphi^{\prime}=0$ при $y=y_{\max }$ привело бы к ошибке, даже когда верхняя граница $y=y_{\max }$ поставлена достаточно далеко. В самом деле, $\varphi^{\prime}$ не стремится к нулю и в пределе $y \rightarrow \infty$, поскольку система проводов (и зарядов на них) бесконечна вдоль направления $x$ [25]. Для самосогласованного расчета $\varphi^{\prime}$ при $y=y_{\max }$ используется методика [26].

До момента зажигания короны плотность ионов равна нулю, $n_{\text {ion }}(x, y, t)=0$, и уравнение (6) дает распределение потенциала в пустом пространстве. Когда у проводов зажигается корона, требуется рассчитать распределение плотности заряда у провода. В двумерных расчетах это обычно делается путем варьирования плотности ионов в первой ячейке у провода $n_{\text {ion }}\left(r_{0}, t\right)[6-8,12]$. Цель этой процедуры - подобрать такое $n_{\text {ion }}\left(r_{0}, t\right)$, чтобы решение системы (4) и (6) удовлетворяло граничному условию $E\left(r_{0}\right)=E_{\mathrm{cor}}$.

Однако, такой способ является очень затратным, поскольку на каждом шаге по времени уравнения (4) и (6) приходится решать несколько раз. Альтернативой является безытерационный подход, подобный примененному в $[1-3,9-11]$. Как показали численные эксперименты, с его помощью можно вести расчет тока с неплохой точностью, так что среднее поле на проводе $E\left(r_{0}\right)$ отличается от $E_{\text {cor }}$ не более, чем на $2 \div 3 \%$.

Пусть требуется найти ток короны $I$ в текущий момент времени $t$. Расчетный цикл начинаем с решения уравнения Пуассона и в результате находим поле на проводе $E_{\mathrm{s}}\left(r_{0}\right)$. Если $E_{\mathrm{s}}\left(r_{0}\right)>E_{\mathrm{cor}}$, то на проводе горит корона, так что в промежуток требуется внедрить заряд $q>0$, который компенсирует превышение $E_{\mathrm{s}}\left(r_{0}\right)$ над $E_{\mathrm{cor}}$.

Заряд $q$ находим из условия

$$
E_{\mathrm{s}}\left(r_{0}\right)-\frac{q}{2 \pi \varepsilon_{0} r_{0}}=E_{\text {cor }}
$$

Переходя к току $I=q / \Delta t$, где $\Delta t-$ шаг по времени, из (9) получаем

$$
I(t)=2 \pi \varepsilon_{0} r_{0} \frac{E_{\mathrm{s}}\left(r_{0}\right)-E_{\mathrm{cor}}}{\Delta t}
$$

Поскольку мы пренебрегаем неоднородностью поля на проводе, то плотность ионного тока, втекающего в первые ячейки сетки, граничащие с проводом, одинакова для всех ячеек и дается формулой $j=I /\left(2 \pi r_{0}\right)$, где $I(t)$ дается формулой (10). Величина $j$ служит граничным условием на проводе для ионного уравнения (4). В момент зажигания короны, формулу (10) можно переписать в дифференциальной форме [11]

$$
I(t)=2 \pi \varepsilon_{0} r_{0} \frac{\mathrm{d} E_{\mathrm{s}}\left(r_{0}\right)}{\mathrm{d} t}
$$

где $\frac{\mathrm{d} E_{\mathrm{s}}\left(r_{0}\right)}{\mathrm{d} t}-$ скорость нарастания поля на поверхности провода.

\section{4. Аналитический расчет потенциала зажигания короны и распределения потенциала, создаваемого зарядами проводов в пустом пространстве}

В системе нескольких параллельных проводов потенциалы зажигания коронных разрядов от каждого провода в общем случае различны. Когда такая система находится во внешнем грозовом поле, коронные разряды у проводов возникают в разные моменты времени. Однако, в бесконечной периодической системе, где все провода пребывают в одинаковых условиях, потенциал зажигания короны одинаков для всех проводов. Его можно рассчитать аналитически. Также аналитически можно найти распределение потенциала $\varphi^{\prime}$, 
создаваемого зарядами периодической бесконечной системы проводов в окружающем провода пространстве.

Исходная формула для потенциала зажигания короны от любого провода из бесконечной периодической системы имеет вид

$$
U_{i}=\frac{q}{4 \pi \varepsilon_{0}} \int_{-\infty}^{+\infty}\left(\frac{\mathrm{d} z}{\sqrt{z^{2}+r_{0}^{2}}}-\frac{\mathrm{d} z}{\sqrt{z^{2}+(2 h)^{2}}}\right)+\frac{q}{4 \pi \varepsilon_{0}} 2 \sum_{k=1}^{\infty} \int_{-\infty}^{\infty}\left(\frac{\mathrm{d} z}{\sqrt{z^{2}+(k D)^{2}}}-\frac{\mathrm{d} z}{\sqrt{z^{2}+(2 h)^{2}+(k D)^{2}}}\right)
$$

где $q=2 \pi r_{0} \varepsilon_{0} E_{\text {cor }}-$ заряд единицы длины провода при напряженности поля $E\left(r_{0}\right)=E_{\text {cor }}$ на его поверхности; $z$ - координата вдоль провода; $k$ - номер провода, отсчитываемый от ближайшего соседа к рассматриваемому. Первый интеграл описывает вклад в потенциал зажигания собственного заряда рассматриваемого провода. Сумма включает вклад всех остальных проводов с двух сторон.

После интегрирования из (12) получаем

$$
U_{i}=\frac{q}{2 \pi \varepsilon_{0}}\left\{\ln \frac{2 h}{r_{0}}+\sum_{k=1}^{\infty} \ln \left[1+\left(\frac{2 h}{k D}\right)^{2}\right]\right\}
$$

Сумму логарифмов в (13) можно точно вычислить. Для этого воспользуемся формулой Эйлера, выражающей гиперболический синус в виде бесконечного произведения [27]

$$
\operatorname{sh} x=x\left(1+\frac{x^{2}}{\pi^{2}}\right)\left(1+\frac{x^{2}}{4 \pi^{2}}\right)\left(1+\frac{x^{2}}{9 \pi^{2}}\right) \cdots,
$$

что можно переписать в виде

$$
\prod_{k=1}^{\infty}\left(1+\frac{a^{2}}{k^{2}}\right)=\frac{\operatorname{sh} \pi a}{\pi a}
$$

где мы положили $x=\pi a=$ const .

Тогда имеем

$$
\sum_{k=1}^{\infty} \ln \left(1+\frac{a^{2}}{k^{2}}\right)=\ln \left[\prod_{k=1}^{\infty}\left(1+\frac{a^{2}}{k^{2}}\right)\right]=\ln \left(\frac{\operatorname{sh} \pi a}{\pi a}\right)
$$

Применяя формулу (15) для вычисления суммы в (13) с $a=2 h / D$, получим выражение для потенциала зажигания короны

$$
U_{i}=\frac{q}{2 \pi \varepsilon_{0}}\left\{\ln \frac{2 h}{r_{0}}+\ln \left[\frac{\operatorname{sh}(\pi 2 h / D)}{\pi 2 h / D}\right]\right\}
$$

Потенциал $U_{i}$ выше, чем потенциал зажигания от уединенного провода. Например, если $D=h=25 \mathrm{~m}, r_{0}=0.7 \mathrm{~cm}$, потенциал зажигания короны от любого провода плоскопараллельной бесконечной системы одинаковых проводов, в которой соседние провода расположены на одинаковом расстоянии, будет в 1.42 раз больше, чем от уединенного провода при прочих равных условиях. Вклад заряда рассматриваемого провода в потенциал $U_{i}$ составляет 70 \% от суммарного вклада всех проводов.

Для проверки сумму в (13) также вычисляли численно. Пока $(2 h / k D)^{2}>0.1$ члены суммы рассчитывали индивидуально. При $(2 h / k D)^{2}<0.1$ раскладывали логарифм и остаточную сумму заменяли интегралом по $k$, который сходится как $1 / k$. Потенциал $U_{i}$ полу- 
чился в 1.39 раз больше, чем потенциал зажигания от уединенного провода, что близко к точному значению 1.42 .

При уменьшении расстояния между проводами $D$ потенциал зажигания $U_{i}$ резко растет, а при увеличении расстояния между проводами потенциал стремится к потенциалу зажигания короны от уединенного провода (рис. 3).

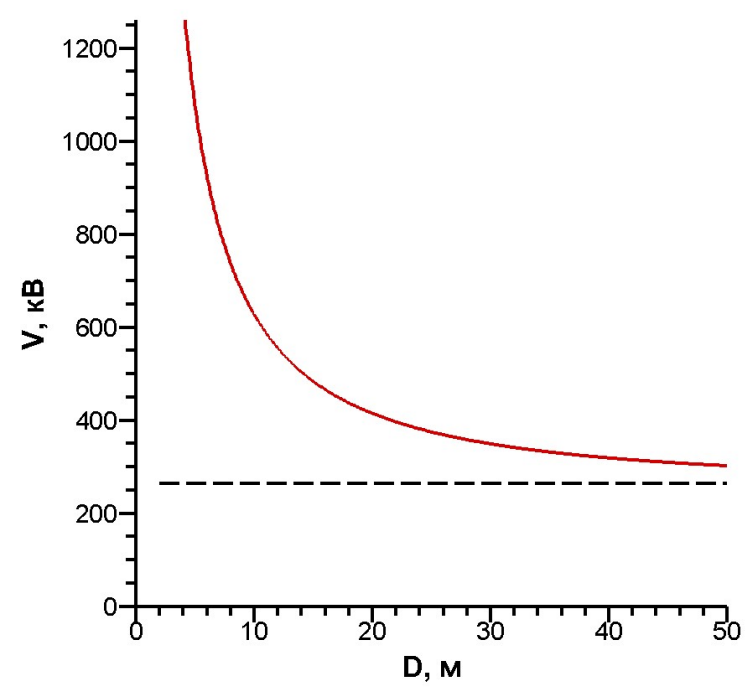

Рис. 3. Зависимость потенциала зажигания короны $U_{i}$ от провода плоскопараллельной бесконечной периодической системы от расстояния между проводами $D$. Высота подвеса проводов $h=25 \mathrm{м}$, радиус проводов $r_{0}=0.7$ см. Пунктирной линией показан потенциал зажигания короны от уединенного провода

Ясно, что в системе конечного числа проводов потенциал зажигания от серединных проводов выше, чем от крайних, т.к. серединные провода подвергается действию ближних зарядов с двух сторон, а крайние - только с одной.

Момент зажигания короны $t_{i}$ в бесконечной системе проводов находится из условия

$$
E_{0}\left(t_{i}\right) h=E_{\text {cor }}\left(r_{0}\right) r_{0}\left\{\ln \left(\frac{2 h}{r_{0}}\right)+\ln \left[\frac{\operatorname{sh}(\pi 2 h / D)}{(\pi 2 h / D)}\right]\right\},
$$

где $E_{0}(t)$ - растущая функция (1) или (2).

Распределение потенциала $\varphi^{\prime}$, индуцированного зарядами проводов в пустом пространстве, найдем из формулы, аналогичной (12), записанной для произвольной точки $x, y$ на плоскости и проинтегрированной по $z$

$$
\begin{aligned}
\varphi^{\prime}(x, y) & =\frac{q}{4 \pi \varepsilon_{0}} \ln \left[\frac{x^{2}+(h+y)^{2}}{x^{2}+(h-y)^{2}}\right]+ \\
& +\frac{q}{4 \pi \varepsilon_{0}} \sum_{k=1}^{\infty} \ln \left\{\left[\frac{(k D-x)^{2}+(h+y)^{2}}{(k D-x)^{2}+(h-y)^{2}}\right] \cdot\left[\frac{(k D+x)^{2}+(h+y)^{2}}{(k D+x)^{2}+(h-y)^{2}}\right]\right\},
\end{aligned}
$$

где $q=2 \pi r_{0} \varepsilon_{0} E\left(r_{0}\right)$ - заряд единицы длины провода. При $x \approx r_{0} \ll h, D$ и $y=h$ выражение (18) для потенциала $\varphi^{\prime}$ сводится к ранее полученной формуле (13) для потенциала на оси любого провода бесконечной системы. 


\section{5. Результаты двумерных расчетов}

Приведем рассчитанное по двумерной программе типичное распределение потенциала $\varphi^{\prime}$, индуцированного зарядами периодической системы проводов в окружающем пространстве в случае, когда внешнее грозовое поле $E_{0}$ меньше грозового поля, в котором от проводов возбуждается корона $E_{0 \text { cor }}$. Этот случай показан на рис. 4. Исследование, которое мы здесь не приводим, показывает, что двумерное численное распределение потенциала $\varphi^{\prime}$ с высокой точностью согласуется с рассчитанным по аналитической формуле (18) (численное решение отклоняется от аналитического на относительную величину не более $1 \div 2 \%$ ). Это говорит о корректности разработанной методики двумерного расчета и созданной компьютерной программы.

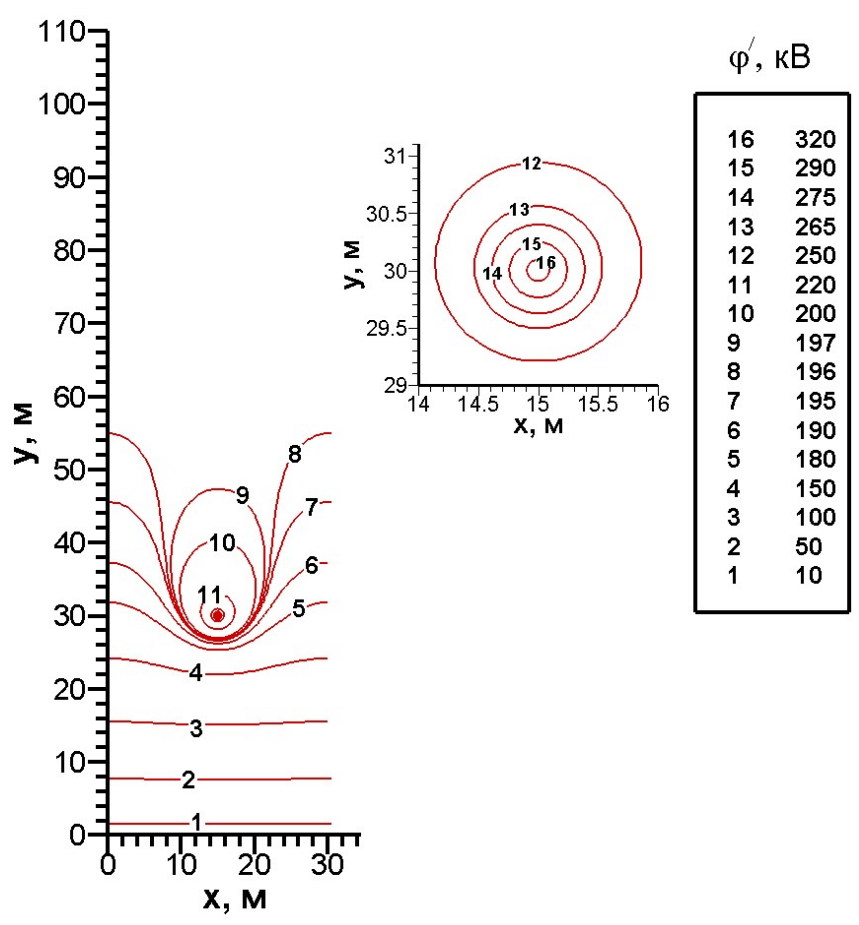

Рис. 4. Изолинии потенциала $\varphi^{\prime}$, создаваемого зарядами плоскопараллельной периодической системы проводов в окружающем пространстве. Высота подвеса проводов $h=30 \mathrm{M}$, расстояние между проводами $D=h=30 \mathrm{м}$, радиус проводов $r_{0}=1 \mathrm{~cm}$, внешнее грозовое поле $E_{0}=130 \mathrm{~B} / \mathrm{cm}$. Пороговое грозовое поле для возбуждения коронного разряда в этих условиях составляет $E_{0 \text { cor }}=168 \mathrm{~B} / \mathrm{cm}$

В ходе увеличения грозового поля у проводов зажигается корона. Временная эволюция погонного тока короны от каждого провода плоскопараллельной периодической решетки проводов дана на рис. 5. Из рис. 5 видно, что чем меньше расстояние между проводами, тем позже зажигается от них корона. Это соответствует формуле (16): при уменьшении $D$ и неизменной высоте подвеса $h$ потенциал зажигания короны повышается.

Ток короны сначала растет со временем, а затем стабилизируется. Амплитуда коронного тока и общее количество внедренного короной заряда на единицу длины провода возрастают с ростом $D$. В момент $t=10 \mathrm{c}$, когда линейный рост грозового поля прекращается, и оно становится постоянным, ток короны начинает резко снижаться. В более поздние моменты времени $t>10 \mathrm{c}$, с увеличением времени ток короны быстро стремится к нулю. Мы видим, что характерный масштаб тока короны с каждого провода составляет $I \sim 1 \mu \mathrm{A} / \mathrm{M}$, что соответствует внедренному короной заряду $Q \sim 5 \mu$ Кл/м за время развития разряда 5 с. 


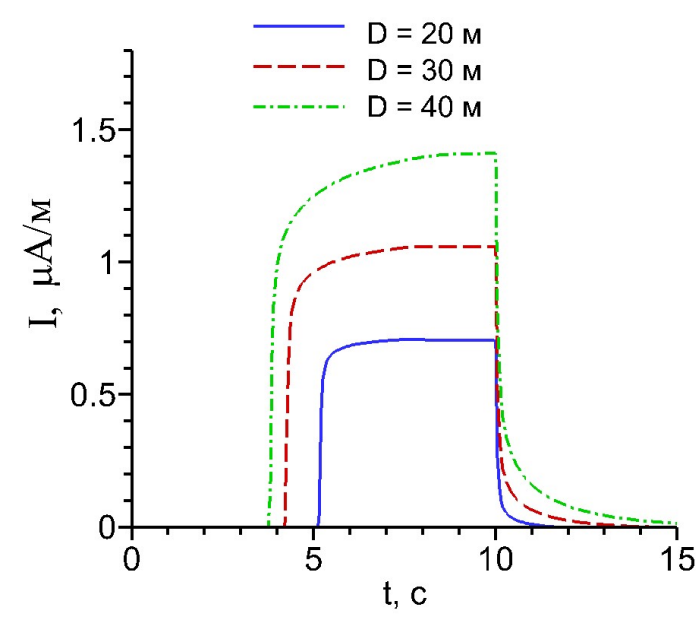

Рис. 5. Зависимости от времени коронного тока от проводов многоэлектродной периодической системы при различных расстояниях между проводами $D$. Высота подвеса проводов $h=30 \mathrm{м}$, радиус проводов $r_{0}=1 \mathrm{~cm}$. Электрическое поле грозового облака растет по линейному закону (1) с $E_{0 \max }=400 \mathrm{~B} \mathrm{cM}^{-1}, \tau=10 \mathrm{c}$

В том случае, когда внешнее грозовое поле растет по релаксационному закону (2) максимальный ток короны с каждого провода имеет тот же порядок величины, что и при линейном нарастании грозового поля. Для проводов с высотой подвеса $h=30$ м и расстоянием между соседними проводами $D=h$ для максимального тока короны расчет дает величину $0.51 \mu \mathrm{A} /$ м. Однако, качественно ход кривой зависимости тока от времени меняется (рис. 6).

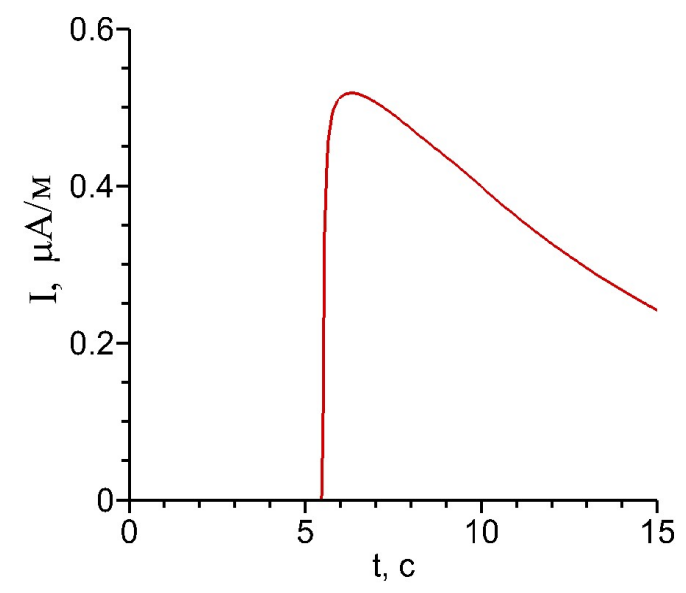

Рис. 6. Зависимость от времени коронного тока от проводов многоэлектродной периодической системы при расстоянии между соседними проводами $D=30$ м. Высота подвеса проводов $h=30 \mathrm{M}$, радиус проводов $r_{0}=1 \mathrm{~cm}$. Электрическое поле грозового облака растет по релаксационному закону (2) с $E_{0 \max }=400 \mathrm{~B} \mathrm{~cm}^{-1}, \tau=10 \mathrm{c}$

Разрядный процесс, ток которого изображен на рис. 5 и 6, соответствует формированию плоского ионного слоя над коронирующими проводами. Этот слой, представляющий собою объединение корон от индивидуальных проводов, был всесторонне изучен в $[9,10]$ на основе одномерной сферической модели короны. Детальное сравнение, которое мы здесь опускаем, показывает, что результаты одномерного и нашего двумерного расчетов тока короны в этом режиме хорошо согласуются друг с другом. 
Рассчитанные по двумерной модели распределения ионов вблизи коронирующих проводов даны на рис. 7-9. Ионные облака сильно вытянуты вверх, поскольку заряды в основном движутся под действием вертикально направленного грозового поля. Сравнение рис. 7-9, соответствующих одинаковому $t=15$ с, показывает, что чем больше расстояние между проводами $D$, тем сильнее корона и выше плотность ионов в данной точке. Это соответствует отмеченному выше возрастанию погонного коронного тока с увеличением $D$.

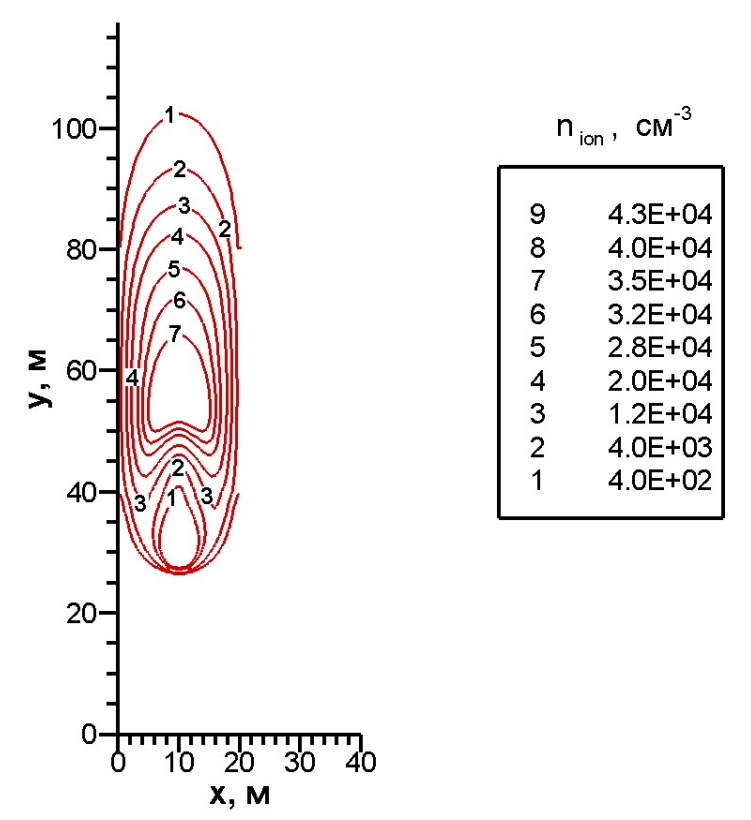

Рис. 7. Распределение плотности ионов, внедренных в окружающее пространство бесконечной периодической системой проводов в момент времени $t=15 \mathrm{c}$. Высота подвеса проводов $h=30$ м, расстояние между проводами $D=20 \mathrm{M}$, радиус проводов $r_{0}=1 \mathrm{~cm}$. Электрическое поле грозового облака растет по линейному закону (1) с $E_{0 \max }=400 \mathrm{~B} \mathrm{~cm}^{-1}, \tau=10 \mathrm{c}$

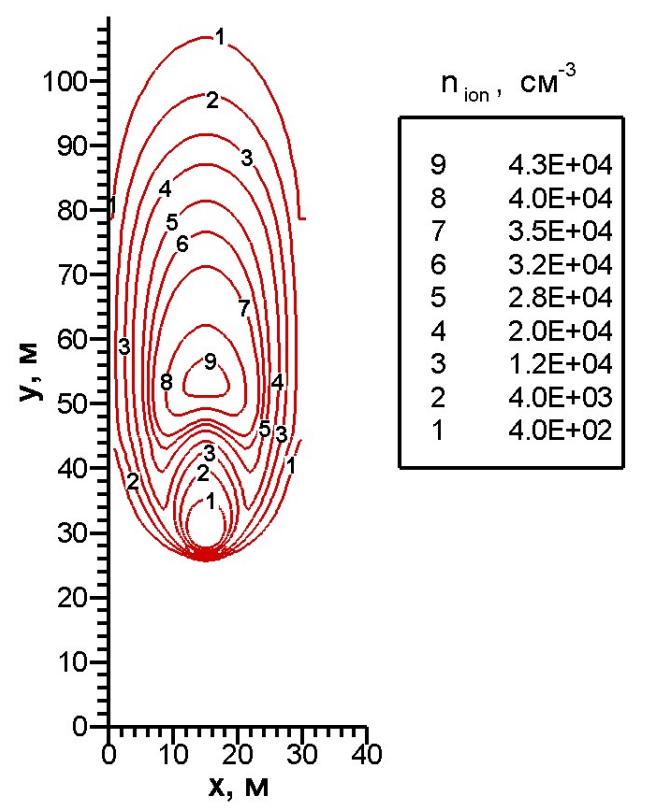

Рис. 8. Распределение плотности ионов, внедренных в окружающее пространство бесконечной периодической системой проводов. Расстояние между проводами $D=30$ м; остальные входные данные - такие же, как на рис. 7 


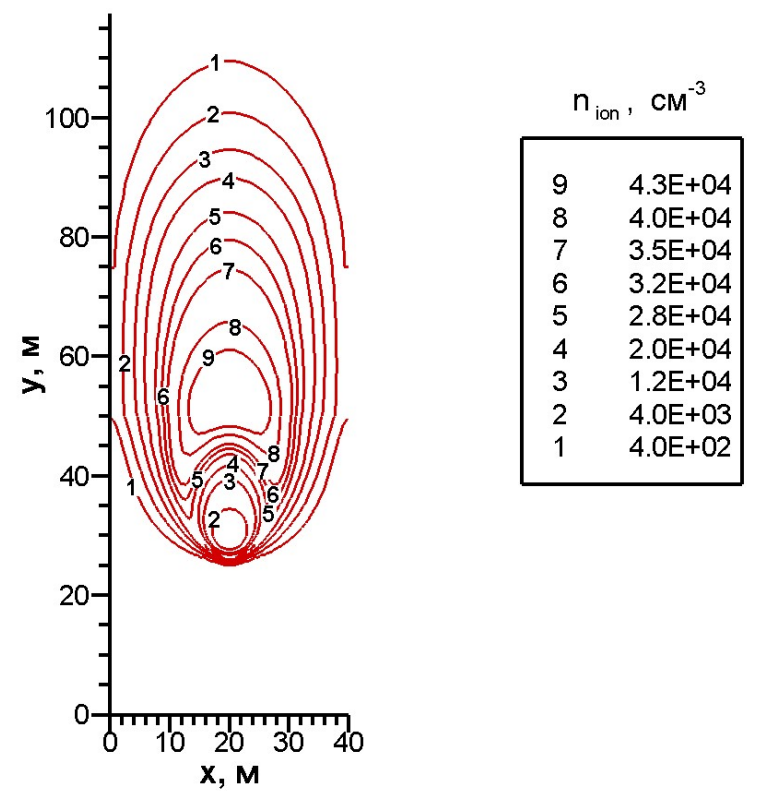

Рис. 9. Распределение плотности ионов, внедренных в окружающее пространство бесконечной периодической системой проводов. Расстояние между проводами $D=40$ м; остальные входные данные - такие же, как на рис. 7 и 8

Подчеркнем, что ионные облака от соседних проводов не могут перекрываться, т.к. силовые линии поля, вдоль которых дрейфуют ионы, никогда не пересекаются. После соприкосновения корон пространственные распределения поля и ионной плотности перестраиваются. Силовые линии поля и траектории ионов в месте сомкнувшихся корон устремляются вертикально вверх. Об этом говорит и рассчитанное распределение потенциала $\varphi^{\prime}$ от системы коронирующих проводов, показанное на рис. 10.
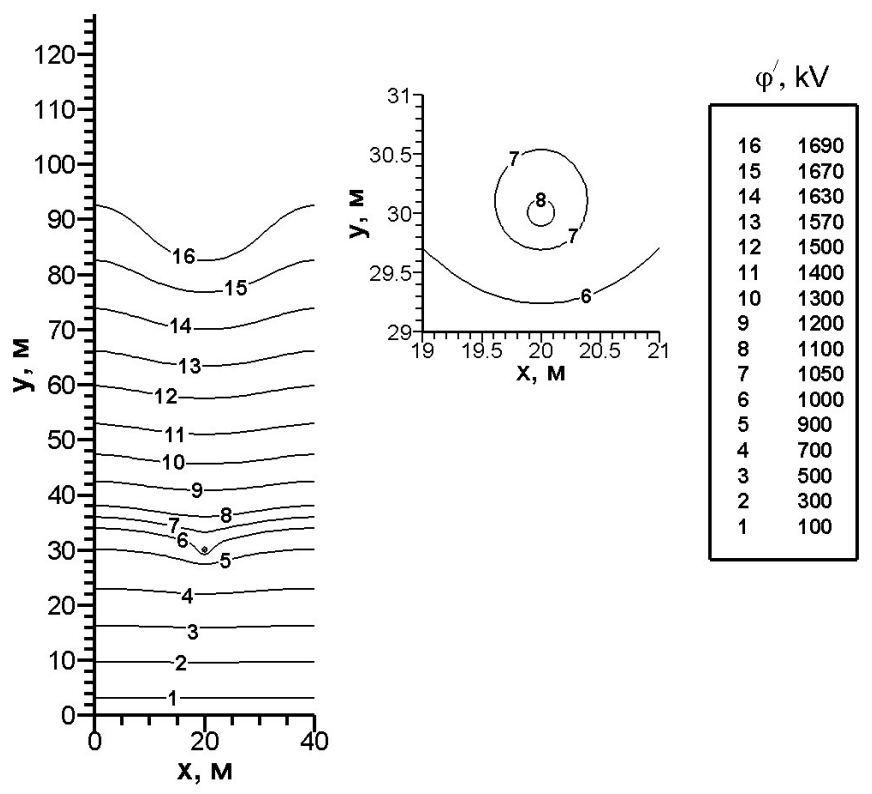

Рис. 10. Линии равного потенциала $\varphi^{\prime}$, индуцированного зарядами проводов и объемным зарядом короны от бесконечной периодической системы проводов в момент времени $t=15 \mathrm{c}$. Высота подвеса проводов $h=30 \mathrm{M}$, расстояние между проводами $D=h=40 \mathrm{м}$, радиус проводов $r_{0}=1 \mathrm{~cm}$. Электрическое поле грозового облака растет по закону линейному закону (1) с $E_{0 \max }=400 \mathrm{~B} \mathrm{cM}^{-1}, \tau=10 \mathrm{c}$ 


\section{6. Заключение}

Разработана и протестирована двумерная компьютерная модель для расчета нестационарного коронного разряда от плоскопараллельной периодической решетки одинаковых заземленных проводов. Провода находятся в свободном пространстве над поверхностью земли во внешнем однородном поле грозового облака.

Порог зажигания короны от каждого провода периодической системы найден аналитически. Он оказывается выше, чем от одиночного провода (при прочих равных условиях). Рассчитан коронный ток с каждого провода, распределения плотности ионного внедренного короной заряда, а также распределение потенциала, индуцированного зарядами проводов и объемным зарядом. Расчеты проведены в условиях, когда соприкасаются слои объемного заряда короны от двух соседних проводов в многопроводной системе.

\section{Благодарности}

Работа М.С. Мокрова поддержана грантом РФФИ № 18-38-00051-мол-а.

\section{Литература}

1. Bazelyan E.M. and Raizer Yu.P. The mechanism of lightning attraction and the problem of lightning initiation by lasers// Phys.-Usp. 2000. Vol. 43. Pp.701-16.

2. Aleksandrov N.L., Bazelyan E.M., Carpenter R.B. Jr, Drabkin M.M. and Raizer Yu.P. The effect of coronae on leader initiation and development under thunderstorm conditions and in long air gaps//J. Phys. D: Appl. Phys. 2001. Vol. 34. Pp. 3256-66.

3. Bazelyan E.M., Raizer Yu.P. and Aleksandrov N.L. Corona initiated from grounded objects under thunderstorm conditions and its influence on lightning attachment//Plasma Sources Sci. Technol. 2008. Vol. 17. Pp. 024015.

4. Rizk F.A.M. Analysis of space charge generating devices for lightning protection: performance in slow varying fields//IEEE Trans. Power Deliv. 2010. Vol. 25. Pp. 1996-2006.

5. Rizk F.A.M. Exposure of overhead conductors to direct lightning strikes: modeling of positive streamer inhibition //IEEE Trans. Power Deliv. 2011. Vol.26. Pp. 1156-65

6. Becerra M. Glow corona generation and streamer inception at the tip of grounded objects during thunderstorms: revisited//J. Phys. D: Appl. Phys. 2013. Vol. 46. Pp.135205

7. Mokrov M.S., Raizer Yu.P. and Bazelyan E.M. Development of a positive corona from a long grounded wire in a growing thunderstorm field//J. Phys. D: Appl. Phys. 2013. Vol. 46. P. 455202.

8. Becerra M. Corona discharges and their effect on lightning attachment revisited: Upward leader initiation and downward leader interception//Atmospheric Research. 2014. Vol. 149. P. 316.

9. Bazelyan E.M., Raizer Yu.P. and Aleksandrov N.L. Non-stationary corona around multi-point system in atmospheric electric field: I. Onset electric field and discharge current//Journal of Atmospheric and Solar-Terrestrial Physics. 2014. Vol. 109. P. 80.

10. Bazelyan E.M., Raizer Yu.P. and Aleksandrov N.L. Non-stationary corona around multi-point system in atmospheric electric field: II. Altitude and time variation of electric field//Journal of Atmospheric and Solar-Terrestrial Physics. 2014. Vol. 109. P. 91.

11. Bazelyan E.M., Raizer Yu.P. and Aleksandrov N.L. The effect of space charge produced by corona at ground level on lightning attachment to high objects//Atmospheric Research. 2015. Vol. 153. Pp. 74-86.

12. Nguyen N.C., Guerra-Garcia C., Peraire J. and Martinez-Sanchez M. Computational study of glow corona discharge in wind: Biased conductor//Journal of Electrostatics. 2017. Vol.89. P.1

13. Golde, R.H.(Ed.), Lightning. New York: Academic. 1977.

14. Rakov V.A. and Uman M.A. Lightning: Physics and Effects. Cambridge: Cambridge University Press. 2003. 
15. Montanyà J., van der Velde O., and Williams E. R. Lightning discharges produced by wind turbines//J. Geophys. Res. Atmos. 2014. Vol. 119. Pp.1455-1462. doi:10.1002/2013JD020225

16. Standler R.B. and Winn W.P. Effects of coronae on electric fields beneath thunderstorms//Quarterly Journal of the Royal Meteorological Society. 1979. Vol. 105. Pp. 285-302.

17. Soula S. and Chauzy S. Multilevel measurement of the electric field underneath a thundercloud. 2. Dynamical evolution of a ground space charge layer//Journal of Geophysical Research. 1991. Vol. 96. Pp. 22327-22336.

18. Soula S. Transfer of electrical space charge from corona between ground and thundercloud: measurements and modeling//Journal of Geophysical Research. 1994. Vol. 99. Pp. 10759-10765

19. Bazelyan E.M. and Raizer Yu.P. Lightning physics and lightning protection. Moscow: Nauka. 2001; Bristol: IOP. 2000.

20. Bazelyan E.M. and Drabkin M.M. Scientific and technical basis for preventing lightning strikes to earth bound objects.// In: Power Engineering Society General Meeting, IEEE,13-17 July. 2003. Vol. 4. Pp. 2201-2208.

21. Гувернюк С.В., Максимов Ф.А. Исследование обтекания решетки цилиндров//Физико-химическая кинетика в газовой динамике. 2019. Т.20, вып. 3. http://chemphys.edu.ru/issues/2019-203/articles/823/

22. Azarenok B.N. Generation of structured difference grids in two-dimensional nonconvex domains using mappings //Comput. Math. Math. Phys. 2009. Vol. 49. Pp.797-809.

23. Ferzinger J.H. and Peric M. Computational Methods for Fluid Dynamics. Berlin: Springer. 2002.

24. Wesseling P. Principles of Computational Fluid Dynamics. Berlin: Springer. 2001.

25. Тамм И.Е. Основы теории электричества М.: Наука. 1989. [Tamm I.E. Fundamentals of the Theory of Electricity. Mir. 1979.]

26. Birdsall C.K. and Langdon A.B. Plasma Physics via Computer Simulation. Taylor \& Francis. 2004. Chapter 14. Pp. 318-322.

27. Лаврентьев М.А., Шабат Б.В. Методы теории функций комплексного переменного. М.: Наука. 1973.

\section{References}

1. Bazelyan, E. M., and Raizer, Yu. P., "The mechanism of lightning attraction and the problem of lightning initiation by lasers," Phys.-Usp., Vol. 43, 2000, pp. 701-16.

2. Aleksandrov, N. L., Bazelyan, E. M., Carpenter, R. B. Jr, Drabkin, M. M., and Raizer, Yu. P., "The effect of coronae on leader initiation and development under thunderstorm conditions and in long air gaps," J. Phys. D: Appl. Phys., Vol. 34, 2001, pp. 3256-66.

3. Bazelyan, E. M., Raizer, Yu. P., and Aleksandrov, N. L., "Corona initiated from grounded objects under thunderstorm conditions and its influence on lightning attachment," Plasma Sources Sci. Technol., Vol. 17, 2008, p. 024015.

4. Rizk, F. A. M., "Analysis of space charge generating devices for lightning protection: performance in slow varying fields," IEEE Trans. Power Deliv., Vol. 25, 2010, pp. 1996-2006.

5. Rizk, F. A. M., "Exposure of overhead conductors to direct lightning strikes: modeling of positive streamer inhibition," IEEE Trans. Power Deliv., Vol. 26, 2011, pp. 1156-1165.

6. Becerra, M., "Glow corona generation and streamer inception at the tip of grounded objects during thunderstorms: revisited," J. Phys. D: Appl. Phys., Vol. 46, 2013, p. 135205.

7. Mokrov, M. S., Raizer, Yu. P., and Bazelyan, E. M., "Development of a positive corona from a long grounded wire in a growing thunderstorm field," J. Phys. D: Appl. Phys., Vol. 46, 2013, p. 455202.

8. Becerra, M., "Corona discharges and their effect on lightning attachment revisited: Upward leader initiation and downward leader interception," Atmospheric Research, Vol. 149, 2014, p. 316. 
9. Bazelyan, E. M., Raizer, Yu. P., and Aleksandrov, N. L., "Non-stationary corona around multi-point system in atmospheric electric field: I. Onset electric field and discharge current," Journal of Atmospheric and Solar-Terrestrial Physics, Vol. 109, 2014, p. 80.

10. Bazelyan, E. M., Raizer, Yu. P., and Aleksandrov, N. L., "Non-stationary corona around multi-point system in atmospheric electric field: II. Altitude and time variation of electric field," Journal of Atmospheric and Solar-Terrestrial Physics, Vol. 109, 2014, p. 91.

11. Bazelyan, E. M., Raizer, Yu. P., and Aleksandrov, N. L., "The effect of space charge produced by corona at ground level on lightning attachment to high objects," Atmospheric Research, Vol. 153, 2015, pp. 74-86.

12. Nguyen, N. C., Guerra-Garcia, C., Peraire, J., and Martinez-Sanchez, M., "Computational study of glow corona discharge in wind: Biased conductor," Journal of Electrostatics, Vol. 89, 2017, p. 1

13. Golde, R. H. (Ed.), Lightning, New York: Academic. 1977.

14. Rakov, V. A., and Uman, M. A., Lightning: Physics and Effects, Cambridge: Cambridge University Press. 2003.

15. Montanyà, J., van der Velde, O., and Williams, E. R., "Lightning discharges produced by wind turbines," J. Geophys. Res. Atmos., Vol. 119, 2014, pp.1455-1462. doi:10.1002/2013JD020225

16. Standler, R. B., and Winn, W. P., "Effects of coronae on electric fields beneath thunderstorms," Quarterly Journal of the Royal Meteorological Society, Vol. 105, 1979, pp. 285-302.

17. Soula, S., and Chauzy, S., "Multilevel measurement of the electric field underneath a thundercloud. 2. Dynamical evolution of a ground space charge layer," Journal of Geophysical Research, Vol. 96, 1991, pp. 22327-22336.

18. Soula, S., "Transfer of electrical space charge from corona between ground and thundercloud: measurements and modeling," Journal of Geophysical Research, Vol. 99, 1994, pp. 10759-10765.

19. Bazelyan, E. M., and Raizer, Yu. P., Lightning physics and lightning protection, Moscow: Nauka. 2001; Bristol: IOP. 2000.

20. Bazelyan, E. M., and Drabkin, M. M., "Scientific and technical basis for preventing lightning strikes to earth bound objects," In: Power Engineering Society General Meeting, IEEE, Vol. 4, July 2003, pp. 2201-2208.

21. Guvernjuk, S. V., Maksimov, F. A., "Issledovanie obtekanija reshetki cilindrov," Fiziko-himicheskaja kinetika v gazovoj dinamike (Physical-Chemical Kinetics in Gas Dynamics), Vol. 20, No. 3, 2019. http://chemphys.edu.ru/issues/2019-20-3/articles/823/

22. Azarenok, B. N., "Generation of structured difference grids in two-dimensional nonconvex domains using mappings," Comput. Math. Math. Phys., Vol. 49, 2009, pp.797-809.

23. Ferzinger, J. H., and Peric, M., Computational Methods for Fluid Dynamics, Berlin: Springer. 2002.

24. Wesseling, P., Principles of Computational Fluid Dynamics, Berlin: Springer. 2001.

25. Tamm, I. E. Osnovy teorii jelektrichestva, Nauka. 1989. [Tamm, I. E., Fundamentals of the Theory of Electricity, Mir. 1979.]

26. Birdsall, C. K., and Langdon, A. B., Plasma Physics via Computer Simulation, Taylor \& Francis. Chapter 14, 2004, pp. 318-322.

27. Lavrent'ev, M. A., Shabat, B. V., Metody teorii funkcij kompleksnogo peremennogo (Methods of the Theory of Functions of a Complex Variable), M.: Nauka. 1973. 